01

\title{
Новый метод описания затухающих колебаний балки с одним заделанным концом
}

\author{
() С.Ш. Рехвиашвили, А.В. Псху
}

Институт прикладной математики и автоматизации КБНЦ РАН, 360000 Нальчик, Россия

e-mail: rsergo@mail.ru

Поступило в Редакцию 17 июля 2018 г.

В окончательной редакции 17 февраля 2019 г.

Принято к публикации 19 марта 2019 г.

Предложен метод описания затухающих колебаний заделанной с одной стороны балки с учетом динамического гистерезиса, который определяет диссипацию механической энергии за счет вязкоупругости. В качестве математической основы использован аппарат дробного интегро-дифференцирования. Проведены экспериментальные исследования быстро затухающих колебаний балки из пенополипропилена. Показано, что теоретическая модель успешно описывает экспериментальные результаты.

Ключевые слова: упругая балка, свободные затухающие колебания, вязкое демпфирование, дробное интегро-дифференцирование, дробный осциллятор.

DOI: $10.21883 /$ JTF.2019.09.48055.284-18

\section{Введение}

Задачи о колебаниях тел с распределенной массой, в частности балок, возникают во многих областях науки и техники - от строительной механики [1] до кремниевой микромеханики и зондовых нанотехнологий [2-5]. Математически эти задачи сводятся к краевым задачам для уравнений в частных производных параболического типа четвертого порядка. Решение этих задач даже без учета реальных внешних источников и полей, которые, как правило, являются нелинейными, представляет собой достаточно трудоемкую математическую процедуру [6-8]. Учет явления вязкоупругости $[9,10]$ в задачах колебаний приводит к дополнительному их усложнению за счет появления в уравнениях интегро-дифференциальных операторов. В данном случае для построения математических моделей эффективно используется аппарат дробного интегро-дифференцирования [11,12].

Для реальных колебательных систем характерно явление динамического гистерезиса (динамической памяти или упругого последействия), которое появляется в результате циклического изменения механических напряжений, максимальная амплитуда которых значительно ниже предела упругости. Динамический гистерезис возникает вследствие диссипации энергии деформации при наличии вязкоупругости и традиционно описывается с точки зрения эквивалентного вязкого или конструкционного демпфирования [8]. Динамический гистерезис может быть также учтен путем введения в уравнение колебаний интегро-дифференциального оператора со степенной функцией памяти в качестве разностного ядра $[9,11]$. В настоящей работе на примере заделанной с одной стороны балки показано, что модель колебаний с дробным интегро-дифференциальным оператором Капуто в целом сопоставима с классической моделью затухающих колебаний с вязким демпфированием и описывает экспериментальные данные. Найдена связь между порядком дробного интегро-дифференцирования и добротностью колебательной системы.

\section{Эффективная масса осциллятора}

Основному тону колебаний балки можно поставить в соответствие колебания эквивалентного точечного осциллятора, который имеет некоторую эффективную массу $m$. Чтобы это показать, воспользуемся методом Рэлея $[8,10]$, который позволяет рассчитать собственную частоту колебаний тела с использованием только кинематических условий и без решения соответствующей краевой задачи для нестационарного уравнения в частных производных.

Потенциальная энергия изогнутой балки, выраженная через момент сил $M(x)$, равна

$$
U=\frac{1}{2 E} \int_{0}^{l} \frac{M^{2}(x)}{f(x)} d x=\frac{k_{\perp}^{2} \Delta z^{2}}{2 E J} \int_{0}^{l} x^{2} d x=\frac{k_{\perp}^{2} \Delta z^{2} l^{3}}{6 E J}
$$

где $k_{\perp}$ - жесткость балки в вертикальном направлении, $E$ - модуль упругости материала балки, $J-$ момент инерции поперечного сечения, $l$ и $\Delta z$ - длина и деформация балки. Приравнивая (1) к потенциальной энергии точечного осциллятора $k_{\perp}^{2} \Delta z^{2} / 2$, получим

$$
k_{\perp}=\frac{3 E J}{l^{3}}=\frac{w E}{4}\left(\frac{h}{l}\right)^{3},
$$

где $J=w h^{3} / 12$ для прямоугольного сечения, $h$ и $w-$ высота и ширина балки. 
Для расчета собственной частоты колебаний методом Рэлея необходимо задать прогиб балки, вызванный сосредоточенной силой $[8,10]$. Функция прогиба заделанной с одной стороны балки может быть представлена в виде

$$
\Delta z(x)=\frac{F_{\perp}}{E J}\left(\frac{l x^{2}}{2}-\frac{x^{3}}{6}\right),
$$

где $F_{\perp}-$ сосредоточенная сила. Потенциальная энергия деформации балки, вычисленная с помощью (3), как это и требуется, совпадает с (1): $U=F_{\perp} \Delta z(l) / 2$. Условная кинетическая энергия колебательного движения балки с учетом функции (3) равна

$$
T=\frac{1}{2} \rho S \frac{F_{\perp}^{2}}{(E J)^{2}} \int_{0}^{l} \Delta z(x) d x=\frac{11}{840} \rho S \frac{F_{\perp}^{2} l^{7}}{(E J)^{2}},
$$

где $\rho$ - плотность материала балки, $S$ - площадь поперечного сечения балки. С использованием (1) и (4) круговая частота основного тона колебаний балки вычисляется по формуле $[8,10] \omega_{0}=\sqrt{U / T}$. Отсюда получаем следующую формулу для собственной частоты колебаний:

$$
f_{0}=\frac{\omega_{0}}{2 \pi} \approx 0.16 \frac{h}{l^{2}} \sqrt{\frac{E}{\rho}}=0.16 \frac{h c_{s}}{l^{2}},
$$

где $c_{s}-$ скорость звука в длинном стержне. Эффективная масса эквивалентного точечного осциллятора есть

$$
m=\frac{k_{\perp}}{\omega_{0}^{2}}=\frac{k_{\perp} T}{U} \approx 0.24 m_{0},
$$

где $m_{0}$ - фактическая масса балки. Формула (6) не содержит момента инерции поперечного сечения балки. Это означает, что данная формула справедлива для балки произвольной формы с постоянным поперечным сечением. Заметим также, что числовой множитель в (6) не является универсальной константой, а зависит от способа закрепления балки.

Метод Рэлея вычисления собственной частоты колебаний обеспечивает достаточно высокую точность; разница с точным выражением, полученным из уравнения свободных колебаний упругой балки с одним заделанным концом [8], обнаруживается только в числовом множителе (5) в третьем знаке после запятой. Эту разницу можно считать несущественной, поскольку погрешность определения модуля упругости различными экспериментальными методами может составлять более $10 \%$.

Таким образом, вместо реальных колебаний заделанной с одной стороны балки можно рассматривать колебания эквивалентного точечного осциллятора, пружинная жесткость и масса которого определяются формулами (2) и (6).

\section{Теоретическая модель}

Рассмотрим сначала линейное дифференциальное уравнение затухающих колебаний точечного осциллятоpa, заданное в безразмерном виде [13]:

$$
\begin{gathered}
\frac{d^{2} u}{d \xi^{2}}+2 D \frac{d u}{d \xi}+u=0, \\
u(\xi)=\frac{x(\xi)}{x_{0}}, \quad \xi=\omega_{0} t, \quad D=\frac{\beta}{\omega_{0}},
\end{gathered}
$$

где $x(\xi)$ - смещение, $t-$ время, $x_{0}-$ начальное смещение, $D$ - безразмерный коэффициент демпфирования, $\beta-$ коэффициент затухания, $\omega_{0}-$ круговая частота колебаний при $\beta=0$, которая определяется формулой (5). К (7) присоединим начальные условия

$$
u(0)=1, \quad u^{\prime}(0)=0 .
$$

Решение задачи Коши (7), (8) имеет вид

$$
\begin{aligned}
u(\xi)= & \exp (-D \xi)\left[\cos \left(\sqrt{1-D^{2}} \xi\right)\right. \\
& \left.+\frac{D}{\sqrt{1-D^{2}}} \sin \left(\sqrt{1-D^{2}} \xi\right)\right] .
\end{aligned}
$$

Укажем на известные недостатки физической модели (7)-(9). Во-первых, согласно (9), полное прекращение колебаний осциллятора происходит при $\xi \rightarrow \infty$, что, очевидно, лишено физического смысла. Во-вторых, хотя и не во всех случаях [14], диссипативная сила, входящая в (7), не описывается принципом наименьшего действия, который применим строго для консервативных систем. Несмотря на эти недостатки, модель (7)-(9) успешно воспроизводит множество натурных экспериментов и часто используется на практике.

Приведем теперь вывод уравнения колебаний осциллятора с дробным интегро-дифференциальным оператором, который учитывает эффект динамической памяти. Для этого запишем уравнение движения в виде

$$
p(x, t)=\int_{0}^{t} G\left(t-t^{\prime}\right) F\left(x, t^{\prime}\right) d t^{\prime},
$$

где $p(x, t)$ - импульс, $F(x, t)-$ сила, $G(t)-$ функция памяти. Функция памяти в (10) определяет изменение импульса механической системы как реакцию на воздействие силы в виде дельта-функции Дирака. Если диссипация в системе отсутствуют, то в качестве функции памяти выступает единичная функция Хевисайда. Это соответствует идеальной памяти в консервативной системе, когда импульс механической системы не изменяется со временем после кратковременного воздействия силы. Наличие диссипации означает, что система постепенно „забывает“ о первоначальном воздействии силы. При этом функция Хевисайда „размывается“, что 
в простейшем случае можно описать двухпараметрической степенной функцией

$$
G(t)=\frac{1}{\Gamma(\alpha)} \frac{b}{\left(\omega_{0} t\right)^{1-\alpha}}, \quad 0<\alpha \leq 1,
$$

где $b-$ положительная константа, $\alpha-$ определяет интенсивность диссипации энергии. При $\alpha=1$ диссипация отсутствует. Подставляя (11) в (10) и обращая дробный интеграл, с учетом формулы для упругой силы $F(x, t)=-k_{\perp} x$ получаем уравнение свободных колебаний так называемого дробного осциллятора:

$$
\begin{gathered}
\frac{d^{1+\alpha} u}{d \xi^{1+\alpha}}+b u=0, \\
\frac{d^{1+\alpha} u(\xi)}{d \xi^{1+\alpha}}=\frac{1}{\Gamma(1-\alpha)} \int_{0}^{\xi} \frac{d^{2} u(s)}{d s^{2}} \frac{d s}{(\xi-s)^{\alpha}} .
\end{gathered}
$$

Интегро-дифференциальный оператор в (12) представляет собой дробную производную Капуто порядка $1+\alpha[12,15]$. Решение задачи Коши (12), (8) имеет вид

$$
\begin{gathered}
u(\xi)=E_{1+\alpha}\left(-b \xi^{1+\alpha}\right), \\
E_{\rho}(z)=\sum_{k=0}^{\infty} \frac{z^{k}}{\Gamma(\rho k+1)}, \quad \rho \geq 0,
\end{gathered}
$$

где $E_{\rho}(z)-$ функция Миттаг-Леффлера. Из свойств функции $E_{\rho}(z)$ следует, что решение (13) имеет конечное число вещественных нулей, зависящее от параметpa $\alpha$ [16-18]. Физически это означает, что в отличие от классического осциллятора с вязким демпфированием затухающие колебания, которые описываются уравнением (12), не имеют бесконечную длительность. При $\xi \rightarrow+\infty$ функция $E_{1+\alpha}\left(-b \xi^{1+\alpha}\right)$, не пересекая вещественную ось, стремится к нулю как степенная функция. В области осцилляций (т.е. в области существования нулей) при значениях $\alpha$, близких к единице, функция Миттаг-Леффлера хорошо приближается формулой [17, теорема 1.1.3]

$$
\begin{aligned}
E_{1+\alpha}\left(-b \xi^{1+\alpha}\right) & \approx \frac{2}{1+\alpha} \exp \left(\xi b^{1 /(1+\alpha)} \cos \left(\frac{\pi}{1+\alpha}\right)\right) \\
& \times \cos \left(\xi b^{1 /(1+\alpha)} \sin \left(\frac{\pi}{1+\alpha}\right)\right),
\end{aligned}
$$

что позволяет качественно сравнивать решения (9) и (13).

Итак, имеются две модели затухающих колебаний осциллятора. В первой модели для учета диссипации в уравнение движения (7) вводится дополнительное слагаемое - сила вязкого трения. Во второй модели диссипация учитывается посредством функции динамической памяти (11) и дробной производной в (12). Требуется сравнить решения (9) и (13) и определить численные значения параметров $D, b$ и $\alpha$, при которых

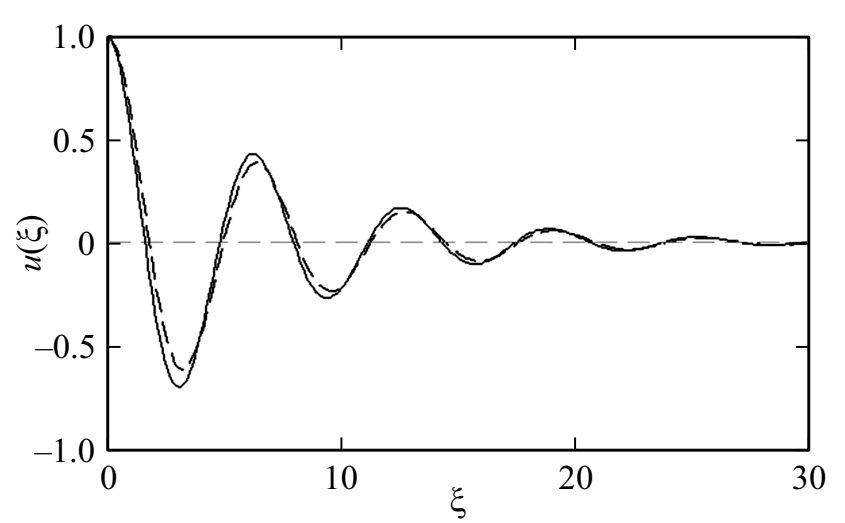

Рис. 1. Пример расчета затухающих колебаний осциллятора: сплошная кривая - формула (13), штриховая кривая формула (9).

эти решения будут максимально приближены друг к другу. Для этого в настоящей работе проводилась оптимизация методом наименьших квадратов по всем трем параметрам. Вычислительный эксперимент показал, что параметр $b$ близок к единице в широком диапазоне изменений параметров $D$ и $\alpha$. Таким образом, было найдено, что в (12) и (13) без существенной потери точности можно принять $b=1$. Оптимизация методом наименьших квадратов по параметрам $D$ и $\alpha$ при $b=1$ показывает, что вместо (13) с приемлемой точностью может быть применена формула (14). Из этого получаются следующие (приближенные) соотношения между параметрами моделей:

$$
\begin{gathered}
\alpha=\frac{\pi}{\operatorname{arcctg}\left(-\frac{\delta}{2 \pi}\right)}-1, \\
\omega=\omega_{0} \sin \left(\frac{\pi}{1+\alpha}\right)=\sqrt{\omega_{0}^{2}-\beta^{2}}, \\
Q=\frac{\pi}{\delta}=\frac{1}{2 D}=\frac{\omega_{0}}{2 \beta},
\end{gathered}
$$

где $\delta$ - логарифмический декремент затухания, $Q-$ добротность, $\omega-$ собственная частота затухающих колебаний. Из проведенного вычислительного эксперимента следует, что при соблюдении соотношений (15)-(17) модель с вязким демпфированием и дробно-дифференциальная модель дают достаточно близкие результаты уже при $Q>3$. На рис. 1 в качестве примера приведены графики быстро затухающих колебаний, которые соответствуют (9) и (13) при $D=0.15$ и $\alpha=0.827$. С увеличением демпфирования осциллятора вплоть до возникновения апериодического движения количественная разница между (9) и (13) возрастает, но качественное соответствие все же сохраняется. Для высокодобротных колебательных систем количественная разница между (9) и (13) при соблюдении соотношений (15)-(17) становится исчезающе малой. В этом случае из (15) и (17) получается простая формула, 
которая связывает порядок дробной производной и добротность: $\alpha=1-2 /(\pi Q)$. Такая же формула получается и при рассмотрении вынужденных колебаний дробного осциллятора [19].

\section{Экспериментальное исследование}

В настоящей работе проводились экспериментальные исследования быстро затухающих колебаний прямоугольной балки из пенополипропилена. Размер балки: длина $l=7.5 \mathrm{~cm}$, ширина $w=5.5 \mathrm{~cm}$, высота $h=7 \mathrm{~mm}$. Принцип работы измерительного стенда заключается в следующем. Балка жестко заделывается с одной выбранной стороны. На край свободной стороны балки приклеивается маленький магнит, масса и размеры которого намного меньше массы и размеров балки. На фиксированном расстоянии от магнита размещается катушка с сердечником, которая служит для детектирования колебаний. Колебания балки возбуждаются механическим способом. Для этого используется электродвигатель с малыми оборотами, к валу которого прикреплена отклоняющая пластина: в процессе вращения эта пластина цепляет образец за свободный край и тем самым возбуждает его колебания. Таким образом, при колебаниях в начальный момент времени обеспечивается определенный профиль изгиба балки, который задается формулой (3). Измерения электрических сигналов осуществлялось с помощью компьютерного генератораосциллографа PCSGU-250.

На рис. 2 показана осциллограмма сигнала. Частота затухающих колебаний исследованного образца и порядок дробной производной в уравнении (12) равны соответственно $f=30.6 \mathrm{~Hz}$ и $\alpha=0.904$. Из этих данных определяются добротность и частота собственных колебаний $Q=6.329$ и $f_{0}=30.7 \mathrm{~Hz}$, а также с использованием формулы (5) оценивается скорость звука в образце пенополипропилена $c_{s}=0.154 \mathrm{~km} / \mathrm{s}$. Полученное значение скорости звука для вспененного полипропи-

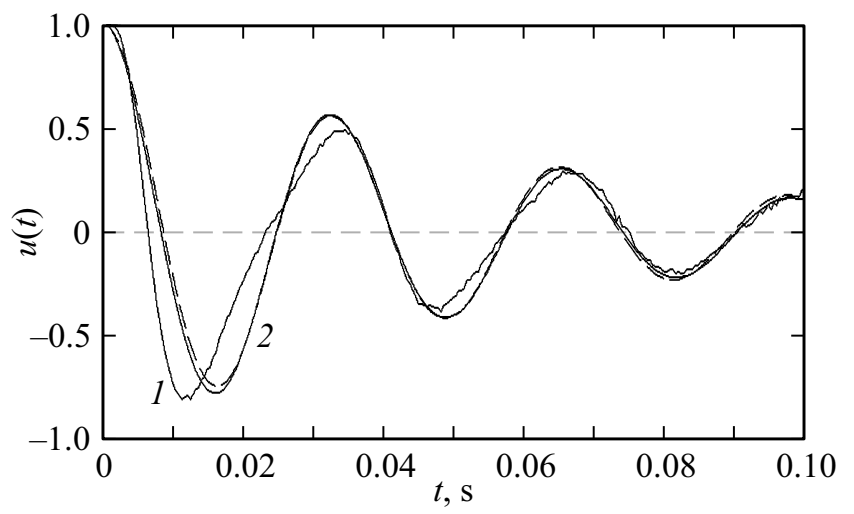

Рис. 2. Осциллограмма сигнала колебаний прямоугольной балки из пенополипропилена с одним заделанным концом: 1 - эксперимент, 2 - расчет (сплошная кривая - формула (13), штриховая кривая - формула (9)). лена оказывается на порядок ниже, чем у различных сплошных образцов [20] и примерно в два раза выше, чем у жидких пен [21].

В нашем случае решения (13) и (9) почти одинаково описывают эксперименты. Несовпадение расчетов с данными экспериментов на начальном участке обусловлено нелинейным характером деформации балки в начальный момент времени. По мере увеличения длительности сигнала эта нелинейность, однако, быстро исчезает, и затухающие колебания хорошо описываются линейными моделями (7) и (12).

\section{Заключение}

В работе предложена новая теоретическая модель для описания затухающих колебаний заделанной с одной стороны балки. Модель основывается на применении математического аппарата дробного интегро-дифференцирования. Дробная производная Капуто в уравнении движения осциллятора (12) учитывает диссипацию энергии; формулы (15)-(17) связывают параметры дробнодифференциальной модели с параметрами классической модели колебаний с вязким демпфированием. Показано, что порядок дробной производной Капуто выражается через логарифмический декремент затухания колебаний.

Из полученных в работе результатов следует, что для изучения диссипативных процессов в различных колебательных физических системах можно использовать дробное диффузионно-волновое уравнение вида [22-24]

$$
\frac{\partial^{1+\alpha} u}{\partial t^{1+\alpha}}-\lambda \Delta u=f(\mathbf{r}, t), \quad 0<\alpha \leq 1
$$

Данное уравнение, в частности, представляется вполне уместной альтернативой телеграфного уравнения в электродинамике. Решение различных начально-краевых задач для диффузионно-волнового уравнения позволит естественным образом учесть диссипативные процессы в задачах механики и электродинамики.

\section{Финансирование работы}

Исследование выполнено при финансовой поддержке РФФИ в рамках научного проекта № 18-51-45005.

\section{Конфликт интересов}

Авторы заявляют, что у них нет конфликта интересов.

\section{Список литературы}

[1] Киселев В.А. Строительная механика. М.: Стройиздат, 1976. $511 \mathrm{c}$.

[2] Butt H.-J., Jaschke M. // Nanotechnology. 1995. Vol. 6. P. 1-7.

[3] Stark R.W., Heckl W.M. // Surf. Sci. 2000. Vol. 457. P. 219-228. 
[4] Rodriguez T.R., Garcia R. // Appl. Phys. Lett. 2002. Vol. 80. P. $1646-1648$.

[5] Korayem M.H., Ebrahimi N., Korayem A.H. // Nanoscience and Nanotechnology. 2011. Vol. 1. N 1. P.14-21.

[6] Коллати, Л. Задачи на собственные значения с техническими приложениями. М.: Наука, 1968. 503 с.

[7] Бидерман В.Л. Теория механических колебаний. М.: Высшая школа, 1980. $408 \mathrm{c.}$

[8] Тимошенко С.П., Янг Д.Х., Уивер У. Колебания в инженерном деле. М.: Машиностроение, 1985. 472 с.

[9] Работнов Ю.Н. Элементы наследственной механики твердых тел. М.: Наука, 1977. 384 с.

[10] Работнов Ю.Н. Механика деформируемого твердого тела. М.: Наука, 1988. 744 с.

[11] Олемской А.И., Флат А.Я. // УФН. 1993. Т. 163. № 12. C. $1-50$.

[12] Уиайкин В.В. Метод дробных производных. Ульяновск: Изд-во Артишок, 2008. 512 с.

[13] Магнус К. Колебания: введение в исследование колебательных систем. / Пер. с нем. М.: Мир, 1982. 304 с.

[14] Шалашов А.Г. // УФН. 2018. Т. 188. № 11. С. 1191-1197.

[15] Псху А.В. Уравнения в частных производных дробного порядка. М.: Наука, 2005. 199 с.

[16] Джррбашян М.М. Интегральные преобразования и представления функций в комплексной области. М.: Наука, 1966. $672 \mathrm{c}$.

[17] Попов А.Ю., Седлецкий А.М. // Современная математика. Фундаментальные направления. 2011. Т. 40. С. 3-171.

[18] Псху А.В. // Мат. заметки. 2005. Т. 77. № 4. С. 592-599.

[19] Псху А.В., Рехвиашвили С.Ш. // Письма в ЖТФ. 2019. T. 45. Вып. 1. С. 34-37.

[20] Полипропилен / Под ред. В.И. Пилиповского, И.К. Ярцева. Л.: Химия, 1967. $316 \mathrm{c.}$

[21] Pierre J., Guillermic R.-M., Elias F., Drenckhan W., Leroy V. // European Phys. J. E. 2013. Vol. 36. P. 113.

[22] Псху А.В. // Изв. РАН. Сер. матем. 2009. Т. 73. № 2. C. $141-182$.

[23] Luchko Y., Mainardi F. // Cent. Eur. J. Phys. 2013. Vol. 11. N 6. P. 666-675.

[24] Luchko Y., Mainardi F. // J. Vib. Acoust. 2014. Vol. 136. N 5. P. 051008 